\title{
Presence Aware Power Saving Mode (PA-PSM) Development for IoT Devices for Energy Conservation
}

\author{
Abdul Saleem, David Jazani \\ Institute for Research in Applicable Computing, University of Bedfordshire, Luton, UK \\ Email: abdul.saleem@study.beds.ac.uk
}

How to cite this paper: Saleem, A. and Jazani, D. (2021) Presence Aware Power Saving Mode (PA-PSM) Development for IoT Devices for Energy Conservation. Open Journal of Energy Efficiency, 10, 81-96. https://doi.org/10.4236/ojee.2021.103006

Received: August 14, 2021

Accepted: September 21, 2021

Published: September 24, 2021

Copyright ( 2021 by author(s) and Scientific Research Publishing Inc. This work is licensed under the Creative Commons Attribution International License (CC BY 4.0).

http://creativecommons.org/licenses/by/4.0/

\section{(c) (i) Open Access}

\begin{abstract}
The smart home is gradually captivating worldwide and provides the best services to human life. It wipes traditional houses by turning them into smart homes; nowadays, numerous energy-efficient devices lead to optimal energy exploitation. It enhances the inhabitant's lifestyle, safety, and comfort since it is suitable for users of diverse incomes and ages. It is essential to exploit the technology to develop energy-efficient smart homes. The design and implementation of energy-efficient smart homes are proposed in this research work. It is a proposed model that can control home appliances, monitor energy usage and send low priority devices in switch OFF mode to save energy. It is a system that uses a combination of a central controller, sensors, modules and PA-PSM algorithm also proposed by this research work. The PIR sensor locates the human within the home and brings the nearby appliance in active mode; however, the rest switch OFF mode, the LDR (light dependent resistor) to sense the intensity of the light to decide if the light is needed else stays in switch OFF mode. The Arduino manages all the sensors, modules, home appliances including scheduling, and energy consumption of individual home appliances. The system automates the process and accepts the commands from end-user through mobile applications to use home appliances. It is a novel approach that has several experiments that help to reduce $20.34 \%$ of energy consumption.
\end{abstract}

\section{Keywords}

Smarthome, BLE, Wi-Fi, Arduino

\section{Introduction}

Energy efficiency in Smart Home Automation has become essential as energy 
crises have become a challenge. Internet of Things (IoT) generally refers to interconnected devices, which consists of a microcontroller, actuators, sensors, and software application to connect, store the sensed data, trigger the alert notification, and share the data with interconnected devices [1]. Energy consumption has been increased significantly worldwide and now leading to the energy crisis. It is necessary to adopt an energy-efficient model to reduce energy consumption; it is achievable through several distinct methods. The sensors can play a vital role to reduce energy consumption. The sensors accumulate the environmental information, send it to the controller to process and act accordingly to switch the appliance OFF [2].

The most superficial meaning of Energy Efficiency is that to utilise less energy to perform the same task by abolishing energy waste. It brings various benefits, significantly reducing the cost to households and the economy [3]. The Buildings are the main area to consume most of the energy, so that developing a building with consideration of energy efficiency will result in an economic gain. Smart home energy management system mainly focuses on monitoring real-time energy consumption and reschedules a particular home appliance's operation or switch OFF to minimise the energy consumption [4] [5] [6]. Energy consumption is increasing worldwide due to population growth, rapid industrialisation, climate change and technological developments, including IoT (Internet of Things) [7]. Energy efficiency brings various benefits, i.e., reducing carbon dioxide emissions, energy imports, and household energy costs [6] [8].

In 2018 , energy consumption globally had already increased more than $250 \%$ since 1990; in the same way, energy consumption in residential accounts has also grown up to $238 \%$ since 1990 with an average $3.57 \%$ yearly increment [9]. Therefore, energy efficiency is vital for smart home automation. In [10] [11] [12] [13], it will be nearly $20 \%$ - $30 \%$ saving energy if energy-efficient smart homes automation is built.

\section{Literature Review}

Several approaches are being applied to numerous research works to reduce energy consumption in smart homes. Shifting appliances from peak to off-peak is one of the most common approaches. It is already in place for decades; however, the profiles system, including the human behaviour aware approach, is also in place.

\subsection{IoT Embedded Smart Energy Management System}

Smart Home Automation with Energy Efficiency refers to a system that supervises and actuates the appliances; the system monitors every appliance's power consumption and suggests reducing energy consumption without losing comfort. The system bases on IoT as shown in Figure 1; therefore have access to all home appliances connected to the system. The IoT Embedded Smart Energy Management System uses sensors and actuators linked to the appliances to sense, collect and send the data to the controller through the communication 
channel. IoT based devices have required a path to connect sensors, actuators and devices to the cloud, and it is possible by a bridge called IoT gateways use to transfer the data, each sensor, actuator, and devise have a unique address to identify within the network. It is the simplest example of a Smart Home Energy Management System (SHEMS). It helps to minimise energy costs to reduce energy bills each month along with the safety, improvement of lifestyle without losing user comfort. Home Energy Management System (HEMS) is a highly integrated system based on real-time sensing, data transmission, and an intelligent processing system [14].

\subsection{Time Based Switching Unit}

Smart Home Energy Management System (SHEMS) is connected with two different power supplies, one is traditional power supplies, and another is the solar system. There is a time-controlled unit connected with the Arduino central controller to set the time of the day; loads within the home are connected through a relay to Arduino. The Blynk mobile app helps for smart switching between power suppliers, i.e., traditional power supply and solar system, so power supply will shift from conventional to solar supply during peak hours to save energy cost as solar supply is cheaper than national power supply per unit cost. It is now common to minimise energy costs by using more inexpensive power supplies than the national power grid or traditional power supply [14].

\section{Energy Management Mechanism}

Energy Management Mechanism (EMM) is responsible for loading the profile on Blynk with the sensed data, and it defines the peak and off-peak hours and switching between loads and sources. It priorities the appliances as per threshold for maximum power consumption of the particular appliances and power consumption. Suppose power consumption exceeds the point, so EMM sends the

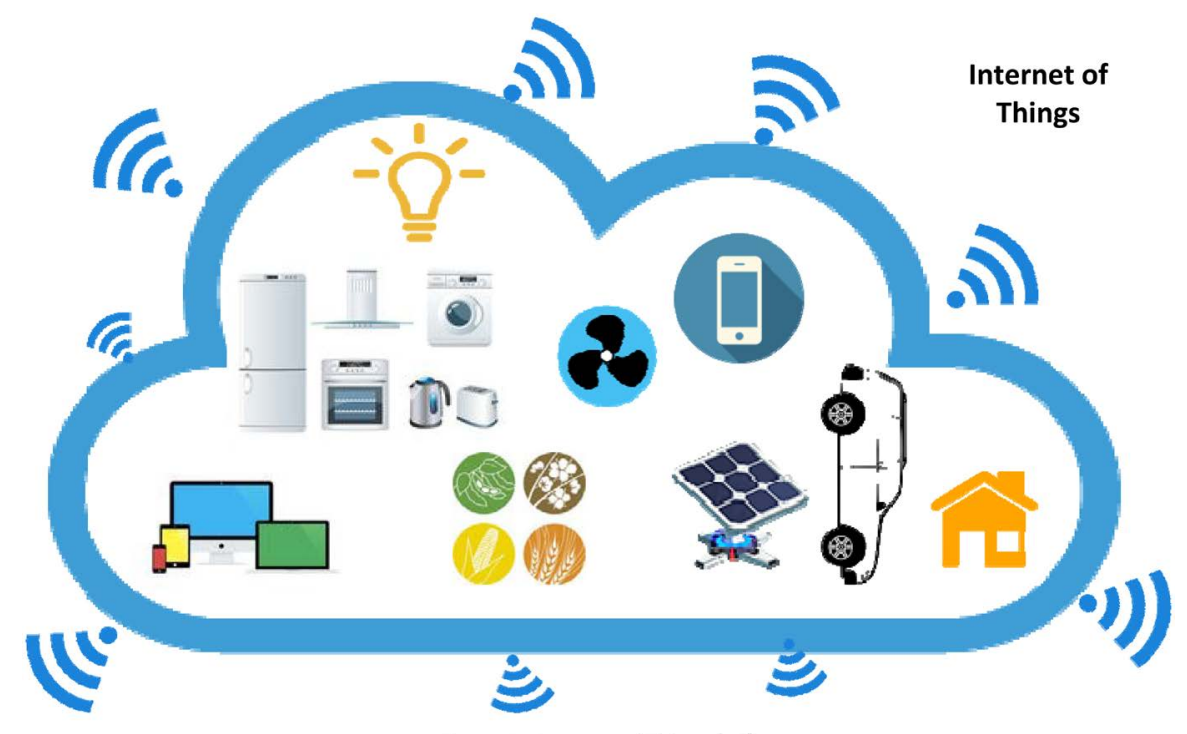

Figure 1. Internet of Things [14]. 


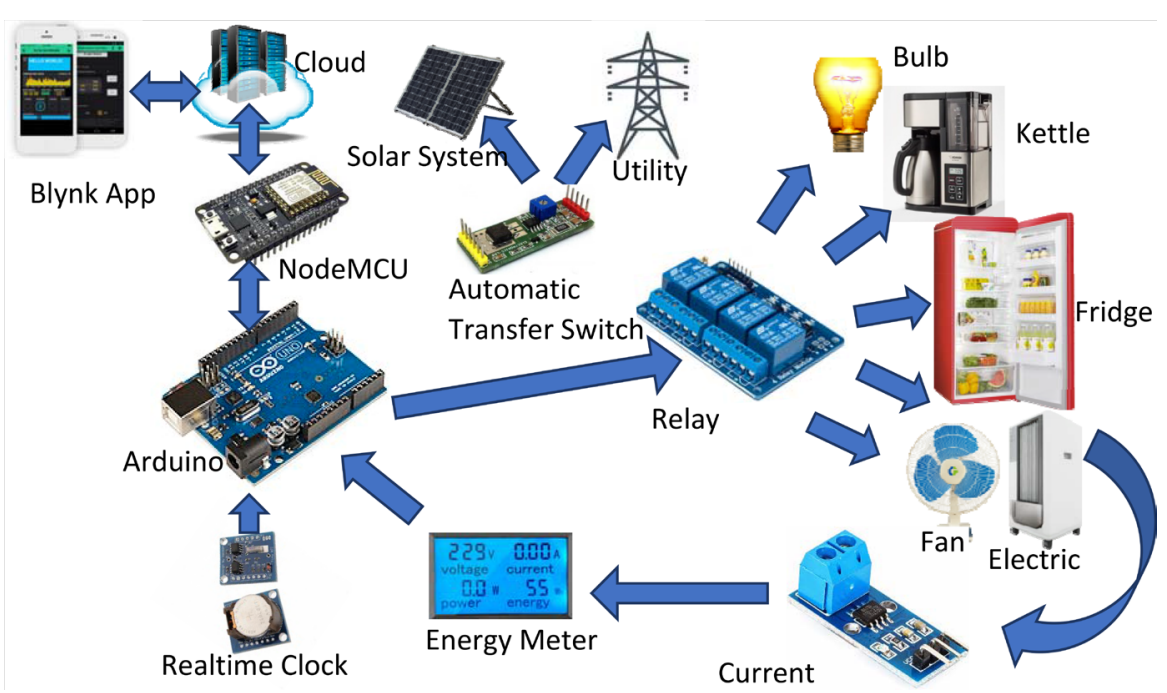

Figure 2. Architecture of smart home energy management system (SHEMS).

least prioritise appliances into the mode switch. It helps to reduce peak energy consumption, so it helps to reduce energy consumption and bring the energy cost down [14].

In [14], energy management is based on peak and off-peak by the use of power grid and renewable solar energy as shown in Figure 2; thus, in peak time load of appliances are managed by the solar system to reduce the electricity cost, renewable energy reduces the electricity cost, however, increase unforeseen cost i.e.

- The initial cost to purchase and install the renewable energy systems is relatively high, and it is including solar panels, wind turbines, wiring, batteries, inverters and, of course, highly skilled professionals to install the system [15].

- Solar systems are highly dependent on sunlight; however, it works during cloudy and rainy weather, but performance moderately dropped significantly in the night-time, it will force users to use traditional costly energy [16].

- Solar energy has to be utilised straightaway or stored into large batteries; these batteries are expensive depends on their storage capacity [17].

- Solar panels need much space on the roof; the more energy they produce, the more space they need, so the top may not be big enough to fit many solar panels [18].

- Pollution related to the solar system is far less than traditional sources of energy, though it generates pollution, primarily, manufacturing of the panels, transportations and installation of the system produces the emission of greenhouse gases [19].

- It is not ideal for the frequent house movers, especially who do not own the house, and it will cause installation and transportation cost once again [20].

\subsection{Human Behaviour Aware - Energy Management System}

Several pieces of research are on the way to address the Energy Management System to reduce energy consumption and reduce the cost involved with it. These methods include using external energy storage, renewable energy supplies, effi- 
cient management of the devices and rescheduling of smart appliances [12]. In Human Behaviour Aware - Energy Management System works based on residential behaviour to decide appliance rescheduling, and the system creates several power profiles based on different household behaviour to see how flexible residents are with rescheduling appliances. This approach can reduce $16 \%$ of the energy usage and $22 \%$ were the peak power-related consumption those rescheduled to off-peak [12].

\subsubsection{Activity Graph}

User activities trigger the events to home appliances that lead to energy consumption, and it is associated actions the user performs to use one or multiple appliances. In contrast, the user in the kitchen might use multiple appliances for a certain period. Activity graph creates blocks and determines the chain of activities performed by the user, and in the next time activity graph model the next activity based on probability. It establishes multiple blocks that depend on the time, user behaviour, type of appliances and usage time [12].

\subsubsection{Flexibility Modelling}

User behaviour on appliances usage can be understood by historical power usage on multiple appliances, years of data has been collected to get closer accuracy of flexibility modelling. It helps create continuous power consumption profiles and smoother profiles; continuous power consumption profiles have an explicit start time for the appliances; however, smoother profiles contain appliances that can be freely distributed across the day [12].

\subsubsection{Behaviour-Aware Scheduling}

Appliance management and rescheduling model based on user behaviour, electricity cost, peak and off-peak power, jobs can be scheduled based on user flexibility, deadlines, electricity prices, peak and off-peak power threshold. This schedule mechanism helps to minimise the energy cost of a house without affecting user comfort, and it is mainly achieved by exploiting the differences in electricity prices between the peak and off-peak energy costs [12].

It is a framework based on household properties such as the number of people, gender, usage behaviour appliances, and user flexibility, and it is based on the historical usage of appliances to reschedule accurately. The framework also set the goals to minimise the electricity cost and peak power consumption. The framework effectively handles the scheduling and can achieve $16 \%$ energy savings and a $22 \%$ reduction in peak power relative cases [12].

In [13] a system based on Human Behaviour - Energy management system entirely based on shifting the load between peaks to the off-peak hour, which may cause discomfort to the home user. This system utilises the energy prices model to adjust the household appliances load between peak and off-peak to minimise electricity cost; however, such models require continuous checking of energy tariff and maintain the load of home appliances; subsequently, it may cause user discomfort within the smart home [21]. 
Some appliances cannot be rescheduled or shifted to another time, i.e., the refrigerator stays switched on to keep food items fresh, so it is essential to keep the fridge on all the time regardless of peak off-peak energy tariff. The refrigerator energy usage is high during the maintenance of required cooling, and after that, it works on low energy usage mode. A television falls in the same category as well; users can turn the TV switch on any time they want; rescheduling the TV will directly hit the user comfort as this cannot be postponed or rescheduled, so these types of appliances must need to require the level of energy all the times to run their operations smoothly [21].

Some appliances can be rescheduled, for instance, a dishwasher. However, if the appliances of this category are already running, they cannot be stopped as this will then cause user discomfort [21]. The heater and air conditioning can also be rescheduled to perform this task. However, Home Energy Management System must hold the information of the required temperature of the house, and it must need to be maintained the home temperature first after that can schedule to off-peak.

\section{Selection and Implementation Process}

Internet of Things has proved to be the most innovative addition in emerging technology and is widely adopted worldwide. They provide the best services in several important areas such as environmental control systems, health systems, transport systems, and Smart Home Automation [22]. However, these devices certainly need the energy to compute their task, and the flood of IoT devices is creating a chaotic environment in terms of increment of energy consumption. Therefore, enormous researches are on the way to reduce the energy consumption for existing devices without losing comfort level [7] [10] [23] [24].

Several wireless technologies help to develop IoT applications. Bluetooth and Wi-Fi are the most popular selection for IoT applications nowadays; Bluetooth Low Energy over Wi-Fi is being widely utilised. The primary aim for BLE is to reduce power consumption, and the recent development of BLE has proved to be the lowest hunger of energy in wireless communication technology. It is mainly selected for IoT development [25] [26] [27]. It is also essential to have a reliable central controller that fulfils the architectural obligation; Arduino microcontroller has been chosen in most of the research [28] [29] due to its flexibility, scalability, energy efficiency, inexpensive, and easy to use as a beginner to intermediate level of programming skills is enough to develop such IoT applications. Arduino controls appliances depend on defined criteria. It is interconnected with sensors and home appliances. The sensors send environmental status to a microcontroller to send command appliances based on defined criteria [30]. Inappropriate selection of microcontrollers may cause poor communication and decrease the performance of the IoT application; therefore, a reliable Arduino is in place. The next challenge is to select appropriate sensors to collect environmental data, i.e. motion detector, light sensor, temperature and humidity 
sensors, and several current sensors attached to each appliance to calculate energy consumption. It is very important to have sensors with durable battery life, energy-efficient and accurate environmental data reading, appropriate sensors with lower battery life, and inaccurate sensor readings will lead the IoT application to collapse [31].

This research proposes an algorithm that plays a vital role in overcoming energy usage in advanced IoT applications for this research work. The algorithm has been named PA-PSM (Presence Aware-Power Saving Mode) as the name explains itself, it detects the motion of human within the defined area, get the intensity of light and get the temperature of the room and let microcontroller to work accordingly, it helps microcontroller to decide to perform the following task such as switch ON/OFF a particular appliance. The proposed algorithm helps to reduce energy consumptions and keep unnecessary home appliances in switch-off mode if no participation is required for the specific device. While the whole IoT system performs its task accordingly, it is wise to learn human behavioural how and when they may use home appliances. It helps predict when and which a particular person could use appliances in the house in the future. It helps to predict and send home appliances in the switch off mode regardless of human makes it home as usage behaviour might suggest that it is out of user behaviour scope, so it is not a time for appliances to be used. It is a successful approach to decrease energy consumption in IoT applications [12] [32] [33].

\subsection{Implementation and Evaluation of PA-PSM}

Two systems have been developed parallel: the non-automation and automation controllers; the scenarios are based on actual homes and offices. There are two different scenarios. The first scenario has no changes and has the same settings and configuration as given by the manufacturer. These devices run with default values and have maximum network traffic as much as the network can support without losing network efficiency.

Scenario 2 has an important task where PA-PSM is implemented; it has the same devices in smart homes as Scenario 1. However, it also works with sensors of human presence finder installed within the house; it is to find human presence within the home and send the report to the controller to bring devices into awake mode if residents are near to smart devices or keep devices sleep mode. This proposed work helps to save the highest level of energy and reduce consumption if devices are being sent to deep sleep mode. It benefits to save up to $20 \%-30 \%$ of the energy consumed by smart devices in-home networks, and it will be significant savings once 75bn devices hit the market by 2025 [34].

\subsection{Non-Automation \& Automation Controllers}

The first step is to bring smart home appliances on a centralised board to control through a microcontroller easily. There are two sets of entire similar boards that have been developed, which are called non-automation and automation. These 
boards have a microcontroller, ESP8266 - ESP01 (a Wi-Fi module), BLE (Bluetooth Low Energy), Load1, Load2, and three sets of current sensors called ACS712. The automation board has some extra features, including HC-SR501 PIR Motion Detector, LDR (Light Dependent Resistor) and DHT 11 Humidity \& Temperature, and a proposed algorithm.

\subsection{Algorithm Flowchart for Automation}

It builds an artefact to aid the microcontroller in deciding when and what situation loads could be sent to switch ON/OFF mode. It is also called an automation algorithm as shown in Figure 3 that defines the rules, and written programming codes act accordingly for the microcontroller to communicate with the sensors and modules. A flow diagram of the automation algorithm is given below.

It initialises the sensors and modules first; after that, the microcontroller checks any devices scheduled. The microcontroller power it up to the scheduled

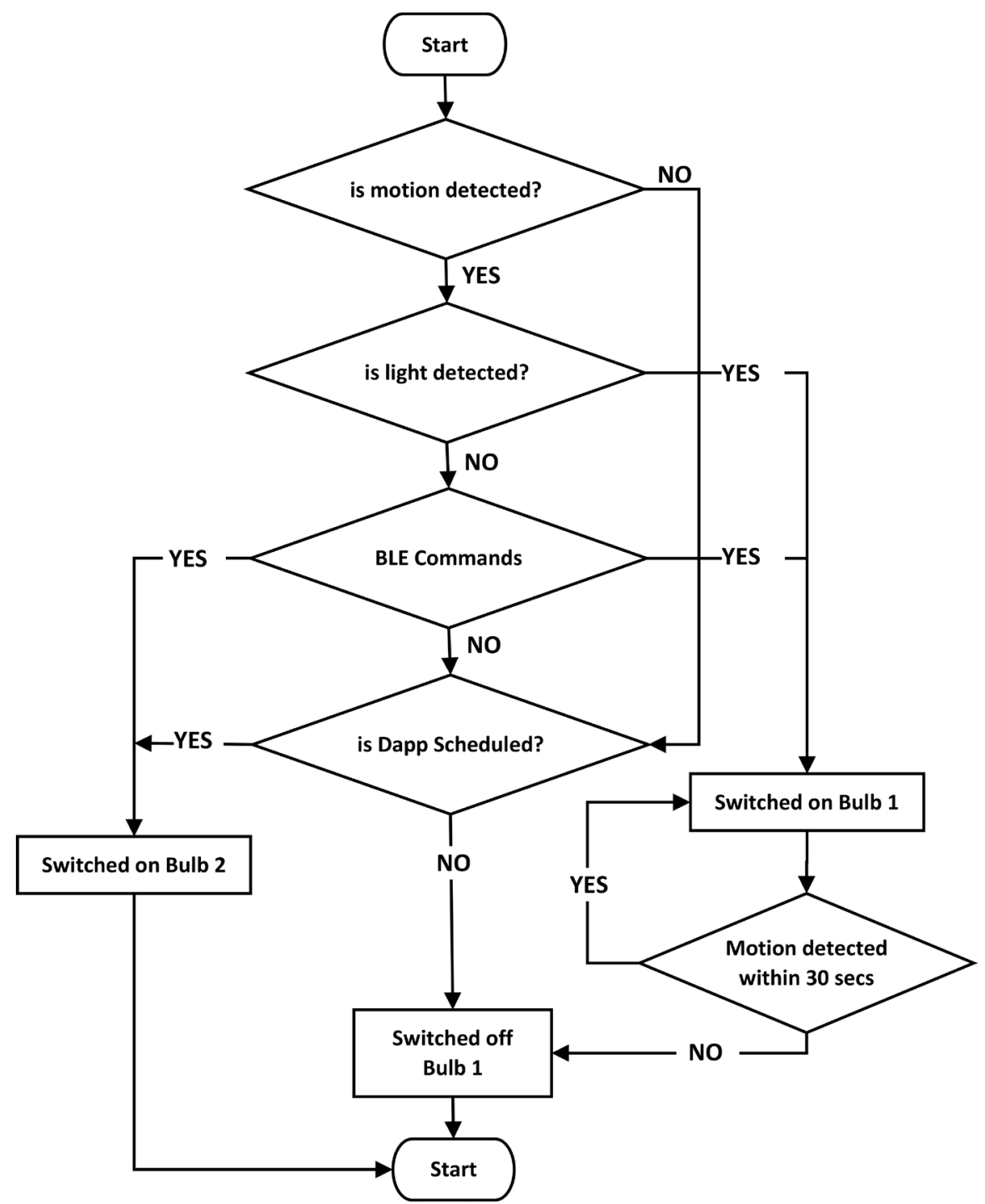

Figure 3. PA-PSM algorithm. 
device, then continue the loop to go to PIR motion detector and starts detecting the motion around, this cycle continues until any motion is detected. Once the activity is detected, it sends the alert to the microcontroller. LDR or Light Dependent Resistor detects whether the light is required; if so, the microcontroller switches ON the load1. Automation algorithm performs the task from several perspectives; for instance, it can power up the loads if motion is detected. If no movement is detected, but the resident connects to it through Bluetooth from a distance, the automation algorithm still works and gets the command from residents mobile apps.

\section{Experiments and Performance Analysis}

There were several experiments have been conducted to achieve the best results from the proposed development. These experiments were held within the home, and sensors were placed on several locations to see how well it performs in distinct places. Therefore, controllers have been placed in several positions within the home one by one. Those places are included the common room, the Television lounge, and the kitchen with a dining area. These are the common places in every home. The residents are usually get together in the mentioned area and mainly in the common room and Television lounge. There were several experiments, but three has been selected. Experiment 1 is given in detail. The rest have been shown as the average of all combined experiments.

\section{Experiment No. 1}

The first "Experiment No 1" was set up in the common area, which may have the highest occupancy ratio compared to other parts of the house. The experiment has been set up on dated 14/04/2021 08:22:53. It has lasted for 168 hours of running time. The automation controller works itself and can detect motions whenever any residents move into the scanning area. Table 1 shows the total consumption for the non-automation controller.

It is noticed that a total of 921.40 watts of energy consumption was recorded for the Non-Automation controller, ESP8266 Wi-Fi controller has the most significant share, up to $53.94 \%$ consumed. It is $8.87 \%$ extra energy consumption as compared to SH-HC-08 Bluetooth Low Energy. In the recent development in IoT devices, including smart home appliances, they are now mainly Wi-Fi. They tend to connect with the internet despite appliances either in any running cycle or not. The Non-Automation controller is installed with two loads on it. Those are controllable by commands through the mobile application. The table below shows the data of both loads on the non-automation controller.

Table 2 holds the information of connected loads; the total running time for the non-automation controller was 168 hours; however, load 1 was ON for 41:28:49, and load 2 was ON for 40:02:17. This controller does not work itself and entirely relies on the resident's manual handling to get the loads to switch ON/OFF by sending commands through a mobile application. Table 2 
Table 1. Experiment 1-Non-Automation Watts Consumption.

\begin{tabular}{cccc}
\hline \multicolumn{4}{c}{ Non-Automation Controller } \\
\hline Device Running Time & Total Watts & SH-HC-08 (BLE) Watts & ESP8266 (Wi-Fi) Watts \\
\hline 0.25 Hour & 1.71 & 0.78 & 0.91 \\
0.50 Hour & 3.40 & 1.54 & 1.83 \\
0.75 Hour & 5.39 & 2.44 & 2.90 \\
1.5 Hours & 10.08 & 4.56 & 5.42 \\
3 Hours & 17.48 & 7.89 & 9.42 \\
6 Hours & 33.34 & 15.03 & 17.98 \\
12 Hours & 66.80 & 30.12 & 36.02 \\
24 Hours & 131.82 & 59.42 & 71.10 \\
48 Hours & 263.96 & 118.99 & 142.37 \\
96 Hours & 525.60 & 236.90 & 283.53 \\
168 Hours & 921.40 & 415.32 & 497.03 \\
\hline
\end{tabular}

Table 2. Experiment No. 1-Non-Automation Loads.

\begin{tabular}{ccc}
\hline \multicolumn{2}{c}{ Non-Automation Controller-Loads } & \\
\hline Device Running Time & Load 1 & Load 2 \\
\hline 0.25 Hour & $00: 10: 40$ & $00: 10: 40$ \\
0.50 Hour & $00: 12: 16$ & $00: 14: 13$ \\
0.75 Hour & $00: 15: 49$ & $00: 21: 51$ \\
1.5 Hours & $00: 17: 16$ & $00: 25: 18$ \\
3 Hours & $00: 43: 09$ & $00: 49: 16$ \\
6 Hours & $01: 48: 43$ & $01: 51: 19$ \\
12 Hours & $04: 52: 26$ & $04: 47: 12$ \\
24 Hours & $07: 55: 39$ & $07: 39: 06$ \\
48 Hours & $13: 06: 00$ & $12: 34: 50$ \\
96 Hours & $24: 29: 38$ & $23: 26: 25$ \\
168 Hours & $41: 28: 49$ & $40: 02: 17$ \\
\hline
\end{tabular}

represents load 1 kept ON for $24.69 \%$, and load 2 kept ON for $23.83 \%$.

The automation Controller has additional modules and sensors on the automation controller board. These additional sensors allow the controller to run in automation mode. The automation controller keeps the home appliances in switch OFF mode until no residents are home. The energy consumption of the Automation Controller has been given in Table 3 Experiment No. 1-Automation Watts Consumption, in the Automation controller 721.51 watts of energy consumed in total 168 hours as compared 921.40 watts in the Non-Automation Controller. The Automation Controller has consumed 21.69\% less energy 
Table 3. Experiment No. 1-Automation Watts Consumption.

\begin{tabular}{cccc}
\hline \multicolumn{3}{c}{ Automation Controller } \\
\hline Device Running Time & Total Watts & SH-HC-08 (BLE) Watts & ESP8266 (Wi-Fi) Watts \\
\hline 0.25 Hour & 1.16 & 0.40 & 0.56 \\
0.50 Hour & 2.26 & 0.77 & 1.10 \\
0.75 Hour & 3.39 & 1.15 & 1.65 \\
1.5 Hours & 6.90 & 2.34 & 3.35 \\
3 Hours & 13.40 & 4.55 & 6.51 \\
6 Hours & 25.73 & 8.73 & 12.50 \\
12 Hours & 51.96 & 17.65 & 25.22 \\
24 Hours & 102.88 & 34.94 & 49.94 \\
48 Hours & 206.30 & 70.10 & 100.11 \\
96 Hours & 411.97 & 139.99 & 199.91 \\
168 Hours & 721.51 & 245.17 & 350.13 \\
\hline
\end{tabular}

Table 4. Experiment No. 1-Automation Loads.

\begin{tabular}{ccc}
\hline \multicolumn{3}{c}{ Automation Controller-Loads } \\
\hline Device Running Time & Load 1 & Load 2 \\
\hline 0.25 Hour & $00: 10: 24$ & $00: 14: 38$ \\
0.50 Hour & $00: 15: 33$ & $00: 19: 47$ \\
0.75 Hour & $00: 21: 52$ & $00: 26: 06$ \\
1.5 Hours & $00: 40: 19$ & $00: 44: 33$ \\
3 Hours & $00: 55: 04$ & $01: 10: 08$ \\
6 Hours & $01: 09: 37$ & $01: 39: 35$ \\
12 Hours & $02: 31: 51$ & $03: 01: 49$ \\
24 Hours & $02: 41: 13$ & $03: 16: 16$ \\
48 Hours & $03: 02: 16$ & $03: 37: 19$ \\
96 Hours & $03: 35: 13$ & $04: 39: 04$ \\
168 Hours & $04: 56: 44$ & $07: 36: 52$ \\
\hline
\end{tabular}

as compared to the Non-Automation controller, in the same way, SH-HC-08 BLE has consumed $40.96 \%$ less energy ESP8266 Wi-Fi is also on the same track and consumed $29.55 \%$ less energy, respectively. However, Automation Controller has been installed with additional sensors, and it is still using far less energy than the non-automation controller.

Table 4 holds that data of Automation controller, load 1 is ready to go into the switch ON mode only if the resident is near the home appliances or receives a command from a resident. The resident can send commands to switch ON/OFF Load 1. However, Load 2 does not depend on a motion detector but can be scheduled, controlled by sending a command from a mobile application. In 168 
hours of running time, Load 1, which is mainly based on a motion detector, was switched $\mathrm{ON}$ for $04: 56: 44$, and that is $2.94 \%$ of the total running time of Experiment No. 1; however, Load 2 was switched on for $07: 36: 52$, and that is $4.53 \%$ of the total running time of Experiment No. 1.

Table 5 summarises all three loads from the non-automation controller, and residents only controlled these loads by connecting through a mobile application. The non-automation controller entirely relies on human interventions to turn the load switch ON/OFF. In the 168 hours running cycle of experiments, the average non-automation loads were 40:44:59 for all three experiments, equalling $24.26 \%$, so $75.74 \%$ of the time, both loads were switched off mode,

Table 5. The Non-automation (Exp1 + Exp2 + Exp3).

\begin{tabular}{cccccc}
\hline \multicolumn{5}{c}{ The Non-Automation (Average of Exp. No 1 + Exp. No 2 + Exp. No 3) } \\
\hline Running Time & Load 1 & Load 2 & Average of Loads & Load ON & Load OFF \\
\hline 0.25 Hour & $00: 09: 25$ & $00: 09: 55$ & $00: 09: 40$ & $64.41 \%$ & $35.59 \%$ \\
0.50 Hour & $00: 16: 09$ & $00: 18: 23$ & $00: 17: 16$ & $57.55 \%$ & $42.45 \%$ \\
0.75 Hour & $00: 24: 58$ & $00: 27: 03$ & $00: 26: 00$ & $57.79 \%$ & $42.21 \%$ \\
1.50 Hours & $00: 55: 32$ & $00: 58: 17$ & $00: 56: 55$ & $63.23 \%$ & $36.77 \%$ \\
3.00 Hours & $01: 42: 09$ & $01: 49: 27$ & $01: 45: 48$ & $58.78 \%$ & $41.22 \%$ \\
6.00 Hours & $02: 49: 23$ & $03: 08: 04$ & $02: 58: 44$ & $49.65 \%$ & $50.35 \%$ \\
12.00 Hours & $05: 02: 30$ & $05: 21: 33$ & $05: 12: 02$ & $43.34 \%$ & $56.66 \%$ \\
24.00 Hours & $09: 41: 08$ & $09: 40: 01$ & $09: 40: 35$ & $40.32 \%$ & $59.68 \%$ \\
48.00 Hours & $16: 31: 21$ & $15: 46: 56$ & $16: 09: 09$ & $33.65 \%$ & $66.35 \%$ \\
96.00 Hours & $26: 36: 24$ & $24: 59: 07$ & $25: 47: 46$ & $26.87 \%$ & $73.13 \%$ \\
168.00 Hours & $41: 20: 47$ & $40: 09: 12$ & $40: 44: 59$ & $24.26 \%$ & $75.74 \%$ \\
\hline
\end{tabular}

Table 6. The Automation (Exp1 + Exp2 + Exp3).

\begin{tabular}{cccccc}
\hline \multicolumn{5}{c}{ The Automation } & Average of Exp. No 1 + Exp. No 2 + Exp. No 3) \\
\hline Running Time & Load 1 & Load 2 & Average of Loads & Load ON & Load OFF \\
\hline 0.25 Hour & $00: 05: 04$ & $00: 05: 58$ & $00: 05: 31$ & $36.76 \%$ & $63.24 \%$ \\
0.50 Hour & $00: 08: 28$ & $00: 08: 38$ & $00: 08: 33$ & $28.50 \%$ & $71.50 \%$ \\
0.75 Hour & $00: 12: 25$ & $00: 12: 29$ & $00: 12: 27$ & $27.67 \%$ & $72.33 \%$ \\
1.50 Hours & $00: 26: 49$ & $00: 26: 53$ & $00: 26: 51$ & $29.83 \%$ & $70.17 \%$ \\
3.00 Hours & $00: 33: 48$ & $00: 37: 29$ & $00: 35: 39$ & $19.80 \%$ & $80.20 \%$ \\
6.00 Hours & $00: 44: 11$ & $00: 52: 50$ & $00: 48: 30$ & $13.47 \%$ & $86.53 \%$ \\
12.00 Hours & $01: 19: 15$ & $01: 33: 00$ & $01: 26: 07$ & $11.96 \%$ & $88.04 \%$ \\
24.00 Hours & $02: 15: 43$ & $02: 30: 58$ & $02: 23: 20$ & $9.95 \%$ & $90.05 \%$ \\
48.00 Hours & $03: 11: 17$ & $03: 31: 54$ & $03: 21: 36$ & $7.00 \%$ & $93.00 \%$ \\
96.00 Hours & $04: 20: 45$ & $04: 56: 35$ & $04: 38: 40$ & $4.84 \%$ & $95.16 \%$ \\
168.00 Hours & $06: 04: 19$ & $07: 05: 15$ & $06: 34: 47$ & $3.92 \%$ & $96.08 \%$ \\
\hline
\end{tabular}


out of 168 hours, loads were in OFF mode for 127:15:01.

Table 6 belongs to the Automation controller. It represents the average of all three experiments, average loads switched ON and OFF time, and their automation loads percentage. The average time for the automation controller was $06: 34: 47$, which is equalled $3.92 \%$. The loads on automation controllers can be controlled by residents instruction through SH-HC-08 BLE commands and depend on the motion detector and if scheduled. The loads were on for only 06:34:47. PA-PSM enabled this research work to save at least $20.34 \%$ of energy compared to the non-automation controller. It enabled the experiments to keep the home appliances in switch OFF mode and managed up to $96.08 \%$ compared to $75.74 \%$ OFF time from the non-automation controller. This research has brought at least $20.34 \%$ of energy savings by implementing the proposed PA-PSM algorithm.

\section{Conclusion}

Researchers around the globe are continuously bringing new smart devices into the market and also paying the most significant attention towards the IoT (Internet of Things), developing the latest devices by enabling Artificial intelligence. This newest storm of smart devices facilitates digital systems to interact with the real environment without human intervention or minimising the human interaction at the lowest level. The researcher of smart appliances believes that the flood of the enormous volume of interconnected devices will produce massive data that will help human society provide the best services to the human world; on the other hand, it will also increase enormous energy consumption. Therefore, it must focus on reducing energy consumption into forthcoming smart devices, and various energy saving methods can achieve it. It is forecasted that nearly 75 billion IoT devices would get interconnected by the year 2025 globally. These devices certainly need a continuous power supply to perform the regular task to provide good services. Therefore, reduction of energy consumptions into existing and forthcoming devices is essential. It is only possible to design a system that can help to reduce energy consumption among interconnected digital objects; therefore, "Presence Aware-Power Saving Mode (PA-PSM) Enhancement for IoT devices for Energy Conservation" has been proposed and implemented to reduce energy consumption, and it has managed at least $20.34 \%$ of energy savings in smart interconnected devices within the smart home network.

\section{Conflicts of Interest}

The authors declare no conflicts of interest regarding the publication of this paper.

\section{References}

[1] Ray, A.K. and Bagwari, A. (2020) IoT Based Smart Home: Security Aspects and Se- 
curity Architecture. 2020 IEEE 9th International Conference on Communication Systems and Network Technologies (CSNT), Gwalior, 218-222. https://doi.org/10.1109/CSNT48778.2020.9115737

[2] Okorie, P.U., Abdu Ibraim, A. and Auwal, D. (2020) Design and Implementation of an Arduino Based Smart Home. 2020 International Congress on Human-Computer Interaction, Optimization and Robotic Applications (HORA), Ankara, 26-27 June 2020, 1-6. https://doi.org/10.1109/HORA49412.2020.9152922

[3] Environmental and Energy Study Institute (2020) Energy Efficiency. https://www.eesi.org/topics/energy-efficiency/description

[4] Leitão, J., Gil, P., Ribeiro, B. and Cardoso, A. (2020) A Survey on Home Energy Management. IEEE Access, 8, 5699-5722.

https://doi.org/10.1109/ACCESS.2019.2963502

[5] Constellation Energy Resources (2019) Energy-Saving Strategies for Smart Homes. https://blog.constellation.com/2019/01/22/smart-home-energy-saving-strategies

[6] Ayan, O. and Turkay, B. (2020) IoT-Based Energy Efficiency in Smart Homes by Smart Lighting Solutions. 2020 21 st International Symposium on Electrical Apparatus \& Technologies (SIELA), Bourgas, 3-6 June 2020, 1-5. https://doi.org/10.1109/SIELA49118.2020.9167065

[7] Jang, H., Lee, T., Kim, S.M., Lee, J. and Park, S. (2020) Energy Storage System Management Method Based on Deep Learning for Energy-Efficient Smart Home. 2020 IEEE International Conference on Consumer Electronics (ICCE), Las Vegas, 4-6 January 2020, 1-2. https://doi.org/10.1109/ICCE46568.2020.9042965

[8] Stimoniaris, D., Foto, H., Voutsakelis, G. and Kokkonis, G. (2020) Design and Construction of HVAC and Lighting Controller with Internet of Things Capabilities. 20203 rd World Symposium on Communication Engineering (WSCE), Thessaloniki, 9-11 October 2020, 84-90. https://doi.org/10.1109/WSCE51339.2020.9275578

[9] IEA (2018) Data and Statistics. IEA. https://www.iea.org/data-and-statistics/?country=WORLD\&fuel=Energy\%20consu mption\&indicator $=$ TotElecCons

[10] Umair, M. and Shah, G.A. (2020) Energy Management of Smart Homes. 2020 IEEE International Conference on Smart Computing (SMARTCOMP), Bologna, 14-17 September 2020, 247-249. https://doi.org/10.1109/SMARTCOMP50058.2020.00054

[11] LI Green (2021) Why Energy Efficiency Is So Important. http://www.ligreen.com/html/why.html https://doi.org/10.5465/AMBPP.2021.10993abstract

[12] Aksanli, B. and Rosing, T.S. (2020) Human Behavior Aware Energy Management in Residential Cyber-Physical Systems. IEEE Transactions on Emerging Topics in Computing, 8, 45-57. https://doi.org/10.1109/TETC.2017.2680322

[13] EPA (2018) Energy Resources for State and Local Governments. EPA-United States-Environmental Protection Agency.

https://www.epa.gov/statelocalenergy/local-energy-efficiency-benefits-and-opportu nities

[14] Ahmad, Z., Abbasi, M.H., Khan, A., Mall, I.S., Khan, M.F.N. and Sajjad, I.A. (2020) Design of IoT Embedded Smart Energy Management System. 2020 International Conference on Engineering and Emerging Technologies (ICEET), Lahore, 22-23 February 2020, 1-5. https://doi.org/10.1109/ICEET48479.2020.9048198

[15] Vourvoulias, A. (2021) Pros and Cons of Solar Energy. Green Match, 23 March 2021.

https://www.greenmatch.co.uk/blog/2014/08/5-advantages-and-5-disadvantages-of- 
solar-energy

[16] E.ON (2020) What Are the Advantages and Disadvantages of Solar Energy? E.ON, 9 June 2020.

https://www.eonenergy.com/spark/solar-energy-advantages-and-disadvantages.htm $\underline{1}$

[17] Solar Guide (2021) Solar Battery Storage: The Best Solar Batteries. Solar GuideTrusted Tradespeople, August 2020.

https://www.solarguide.co.uk/solar-batteries\#

[18] Lukecove (2020) 12 Advantages \& 8 Disadvantages of Solar Panels. September 2020. https://lightningsolar.com.au/comparative-guide-advantages-disadvantages-of-solar -panels

[19] Brandon, M. (2021) 5 Disadvantages of Solar Energy-2021 Guide. Scholarlyoa, January 2021. https://scholarlyoa.com/disadvantages-of-solar-energy

[20] Kay, A. (2020) Solar Advantages and Disadvantages-Should You Get Solar Panels Installed? Greener Energy Group, October 2020.

https://greenerenergygroup.co.uk/blog/solar-advantages-and-disadvantages

[21] Khan, M., Seo, J. and Kim, D. (2020) Real-Time Scheduling of Operational Time for Smart Home Appliances Based on Reinforcement Learning. IEEE Access, 8, 116520 116534. https://doi.org/10.1109/ACCESS.2020.3004151

[22] Stoyanova, M., Nikoloudakis, Y., Panagiotakis, S., Pallis, E. and Markakis, E.K. (2020) A Survey on the Internet of Things (IoT) Forensics: Challenges, Approaches, and Open Issues. IEEE Communications Surveys \& Tutorials, 22, 1191-1221. https://doi.org/10.1109/COMST.2019.2962586

[23] Rehman, A.U., Tito, S.R., Ahmed, D., Nieuwoudt, P., Lie, T.T. and Vallès, B. (2020) An Artificial Intelligence-Driven Smart Home Towards Energy Efficiency: An Overview and Conceptual Model. 2020 FORTEI-International Conference on Electrical Engineering (FORTEI-ICEE), Bandung, 23-24 September 2020, 47-52. https://doi.org/10.1109/FORTEI-ICEE50915.2020.9249816

[24] Kaippilly Radhakrishnan, K., Chinh, H.D., Gupta, M., Panda, S.K. and Spanos, C.J. (2020) Context-Aware Plug-Load Identification toward Enhanced Energy Efficiency in the Built Environment. IEEE Transactions on Industry Applications, 56, 67816791. https://doi.org/10.1109/TIA.2020.3016621

[25] Badihi, B., Sheikh, M.U., Ruttik, K. and Jäntti, R. (2020) On Performance Evaluation of BLE 5 in Indoor Environment: An Experimental Study. 2020 IEEE 31 st Annual International Symposium on Personal, Indoor and Mobile Radio Communications, London, 31 August-3 September 2020, 1-5. https://doi.org/10.1109/PIMRC48278.2020.9217132

[26] Shan, G. and Roh, B.-H. (2020) Performance Model for Advanced Neighbor Discovery Process in Bluetooth Low Energy 5.0-Enabled Internet of Things Networks. IEEE Transactions on Industrial Electronics, 67, 10965-10974. https://doi.org/10.1109/TIE.2019.2962401

[27] Ding, M., et al. (2021) A Bluetooth 5 Transceiver with a Phase-Tracking RX and Its Corresponding Digital Baseband in 40-nm CMOS. IEEE Journal of Solid-State Circuits, 56, 254-266. https://doi.org/10.1109/JSSC.2020.3005788

[28] Vivek, P.S., Rahul, P.V.S., Dyuthy, E.S. and Yadav, S. (2021) Arduino Based Smart System for Control and Effective Billing. 2021 International Conference on Artificial Intelligence and Smart Systems (ICAIS), Coimbatore, 25-27 March 2021, 17061709. https://doi.org/10.1109/ICAIS50930.2021.9395750

[29] Lulla, G., Kumar, A., Pole, G. and Deshmukh, G. (2021) IoT Based Smart Security 
and Surveillance System. 2021 International Conference on Emerging Smart Computing and Informatics (ESCI), Pune, 5-7 March 2021, 385-390.

https://doi.org/10.1109/ESCI50559.2021.9396843

[30] Ismail, N.N., Binti Mustafa, S.Z., Yunus, F. and Abd Warif, N.B. (2020) Internet of Things (IoT) Smart Rubber Scale (SRS) System Using Arduino Platform. 2020 IEEE International Conference on Automatic Control and Intelligent Systems (I2CACIS), Shah Alam, 20 June 2020, 45-50. https://doi.org/10.1109/I2CACIS49202.2020.9140209

[31] Bhartiya, G. and Pathak, P. (2020) Intelligent Lighting Control and Energy Management System. 2020 International Conference on Power Electronics \& IoT Applications in Renewable Energy and Its Control (PARC), Mathura, 28-29 February 2020, 86-89. https://doi.org/10.1109/PARC49193.2020.236563

[32] Sardianos, C., et al. (2020) Real-Time Personalised Energy Saving Recommendations. 2020 International Conferences on Internet of Things (iThings) and IEEE Green Computing and Communications (GreenCom) and IEEE Cyber, Physical and Social Computing (CPSCom) and IEEE Smart Data (SmartData) and IEEE Congress on Cybermatics (Cybermatics), Rhodes, 2-6 November 2020, 366-371.

https://doi.org/10.1109/iThings-GreenCom-CPSCom-SmartData-Cybermatics50389.2 $\underline{020.00072}$

[33] Ooi, B.-Y. and Shirmohammadi, S. (2020) The Potential of IoT for Instrumentation and Measurement. IEEE Instrumentation \& Measurement Magazine, 23, 21-26. https://doi.org/10.1109/MIM.2020.9082794

[34] Rembert, L.F. (2020) Connected Devices Will Generate 79 Zettabytes of Data by 2025. IoT Business News.

https://iotbusinessnews.com/2020/08/10/08984-connected-devices-will-generate-79 -zettabytes-of-data-by-2025 